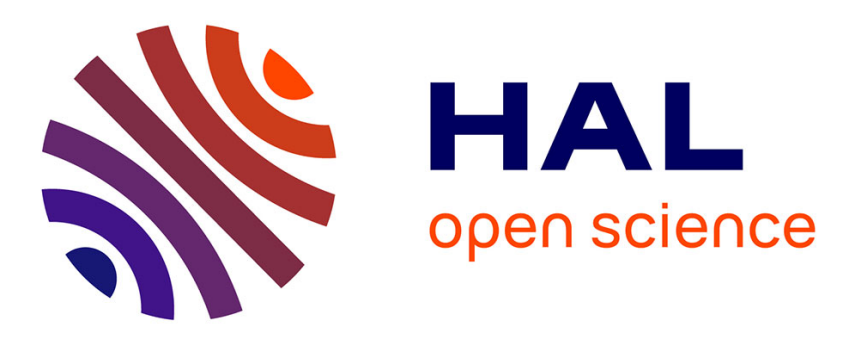

\title{
Depletion layers in polymer solutions: influence of the chain persistence length
}

D. Ausserré, H. Hervet, F. Rondelez

\section{To cite this version:}

D. Ausserré, H. Hervet, F. Rondelez. Depletion layers in polymer solutions: influence of the chain persistence length. Journal de Physique Lettres, 1985, 46 (19), pp.929-934. 10.1051/jphyslet:019850046019092900 . jpa-00232920

\section{HAL Id: jpa-00232920 https://hal.science/jpa-00232920}

Submitted on 1 Jan 1985

HAL is a multi-disciplinary open access archive for the deposit and dissemination of scientific research documents, whether they are published or not. The documents may come from teaching and research institutions in France or abroad, or from public or private research centers.
L'archive ouverte pluridisciplinaire HAL, est destinée au dépôt et à la diffusion de documents scientifiques de niveau recherche, publiés ou non, émanant des établissements d'enseignement et de recherche français ou étrangers, des laboratoires publics ou privés. 
Classification

Physics Abstracts

$36.20 \mathrm{E}-68.45$

\title{
Depletion layers in polymer solutions : influence of the chain persistence length
}

\author{
D. Ausserré, H. Hervet and F. Rondelez \\ Physique de la Matière Condensée (*), Collège de France, \\ 11, place Marcelin-Berthelot, 75231 Paris Cedex 05, France
}

(Reçu le 15 avril 1985, accepté sous forme définitive le 16 août 1985)

\begin{abstract}
Résumé. - Nous proposons un modèle simple permettant de décrire le profil de concentration dans une solution diluée de chaînes semi-flexibles au voisinage d'une paroi solide non-adsorbante. Notre approche est basée sur l'hypothèse d'une concentration de surface non nulle due à la rigidité partielle de la chaîne. Le profil de concentration $\Phi_{\mathrm{SF}}(Z)$ est obtenu à partir de celui des chaînes flexibles $\Phi_{\mathrm{F}}(Z)=\Phi \tanh ^{2}\left(Z / R_{\mathrm{G}} \sqrt{2}\right)$ à condition d'introduire une longueur d'extrapolation $D$ proportionnelle à la longueur de persistance $q$ de la chaîne. Nous montrons que $\Phi_{\mathrm{SF}}(Z)=\Phi_{\mathrm{b}} \tanh ^{2}[(Z+$ $\left.D) / R_{\mathrm{G}} \sqrt{2}\right]$ avec $R_{\mathrm{G}}$ rayon de gyration et $\Phi_{\mathrm{b}}$ concentration en volume. Cette formule décrit très bien des résultats expérimentaux récents obtenus par la méthode de Fluorescence Induite par Ondes Evanescentes, sur des solutions de xanthane, un polysaccharide hydrosoluble.
\end{abstract}

Abstract. - We propose a simple model for the concentration profile induced by a non-adsorbing solid wall in dilute solutions of semi-flexible chains. Our approach is based on the assumption that the restriction in the chain local curvature of the chain creates a non-zero surface concentration. The monomer concentration profile $\Phi_{\mathrm{SF}}(Z)$ is deduced from the mean-field model for flexible chains, $\Phi_{\mathrm{F}}(Z)=\Phi_{\mathrm{b}} \tanh ^{2}\left(Z / R_{\mathrm{G}} \sqrt{2}\right)$, by introducing an extrapolation distance $D$ proportional to the persistence length $q$. We show that $\Phi_{\mathrm{SF}}(Z)=\Phi_{\mathrm{b}} \tanh ^{2}\left[(Z+D) / R_{\mathrm{G}} \sqrt{2}\right]$ where $R_{\mathrm{G}}$ is the chain radius of gyration and $\Phi_{\mathrm{b}}$ is the bulk polymer concentration. This analytical expression provides a very good fit with recent experimental data obtained by the evanescent wave induced fluorescence method (EWIF) in aqueous xanthan solutions.

Polymer solutions exhibit unique interfacial properties which can be utilized in a host of potential applications, ranging from biology to the petroleum recovery [1]. However, owing to the experimental difficulties involved in measurements of monomer concentration profiles in very shallow interfacial regions, quantitative investigations have not been performed until recently. In one previous report [2] we have shown how the newly developed evanescent wave induced fluorescence (EWIF) technique [3] can be used to probe depletion layers at the interface between a non-adsorbing fused silica wall and dilute xanthan solutions. Xanthan was selected because it is a semi-flexible polysaccharide chain of high molecular weight $\left(\bar{M}_{\mathrm{w}}=2 \times 10^{6}\right)$,

$\left(^{*}\right)$ Unité Associée au C.N.R.S. : UA 792. 
the properties of which have been accurately measured by Muller et al. [4] under conditions similar to ours. Because of the large chain persistence length $q=50 \mathrm{~nm}$, the end-to-end distance $\left\langle L^{2}\right\rangle^{1 / 2}$ is $420 \mathrm{~nm}$, i.e. one order of magnitude greater than the expected value for a flexible coil of equivalent mass [5]. This makes the interfacial layer much thicker and the experiments become easier to perform. However the drawback was that the theory for the monomer concentration profile $\Phi(Z)$ had not yet been worked out for semi-flexible chains. Therefore the experimental data could only be compared with the models established in the two opposite cases of fully flexible or fully rigid polymer. The mean-field model of Joanny, Leibler and de Gennes [6] predicts that, for flexible coils, the depletion layer profile in the semi-dilute regime should vary as [3]

$$
\Phi_{\mathrm{F}}(Z)=\Phi_{\mathrm{b}} \tanh ^{2}\left(\frac{Z}{\xi \sqrt{2}}\right)
$$

where $Z$ is the distance normal to the wall. The $F$ subscript in $\Phi_{F}$ refers to the flexible case. $\xi$ is the bulk correlation length and is a decreasing function of the bulk polymer concentration $\Phi_{\mathbf{b}}$. By extension, we expect a similar law to hold in the dilute regime if we replace $\xi$ by the chain radius of gyration $R_{\mathrm{G}}$. On the other hand, the statistical model of Auvray [7] deals with rigid rods of length $L$ and negligible width and yields the following profile :

$$
\Phi_{\mathrm{R}}(Z)=\Phi_{\mathrm{b}} \frac{Z}{L}\left(1-\ln \frac{Z}{L}\right)
$$

independent of the bulk polymer concentration, at least in the isotropic phase. The $R$ subscript in $\Phi_{R}$ refers to the rigid case.

Both models were compared with the experimental results in dilute solutions and only in the second case could a satisfactory agreement be reached. The best fit was achieved for a rod length $L$ of $600 \pm 20 \mathrm{~nm}$, which is actually consistent with the known end-to-end distance of the xanthan chain. It seems a little risky however to describe a chain of contour length $L_{\mathrm{c}}=$ $1800 \mathrm{~nm}$ [4] by an " equivalent rod » of length $600 \mathrm{~nm}$. In other words, since the persistence length $q$ is $50 \mathrm{~nm}$, there are about 18 statistical units (of Kuhn length $2 q$ ) along the chain backbone [8] and the chain must, therefore, retain some flexibility. The use of the rigid rod model is thus a vast over-simplification. Further evidence for this is the experimentally observed dependence of the mean thickness $e$ of the depletion layer on varying the bulk polymer concentration [9]. Such a behaviour is quite contrary to the Auvray model which predicts that $e$ should remain of the order of $L$, independent of $\Phi_{\mathrm{b}}$, as long as the transition to a nematic liquid crystal lyotropic solution is not reached. Therefore we also tried a slightly improved approach in which xanthan was considered as a rod over a distance to the wall equal to twice the persistence length, and as a flexible coil at all larger distances. The concentration profile given by the rod model was used for $Z<2 q$ while a $\tanh ^{2}(Z)$ profile was assumed for $Z>2 q$. This mixed « rigid then flexible " model quantitatively describes the experimental curves, yielding a best fit value of $80 \mathrm{~nm}$ for $q$ and an end-to-end distance of $440 \mathrm{~nm}$. Both of these values appear very reasonable. Moreover the model has the advantage of readily explaining the dependence of $e$ on $\Phi_{\mathrm{b}}$ since $e$ should be comparable to $\xi$, and should thus scale as $\Phi_{b}^{-3 / 4}$. However it suffers from an unphysical discontinuity in $\Phi(Z)$ at $Z=2 q$ and also lacks rigorous theoretical support.

In view of the above situation, we have looked for a more rigorous model which could be valid for semi-flexible chains near a non-adsorbing wall. Our approach is based on the notion of a virtual wall, separated from the real interface by an extrapolation distance related to the actual chain persistence length. We then show that the monomer concentration profile for. semiflexible chains can be naturally deduced from the one already known for flexible coils. Finally, a quantitative comparison is made with the previously published experimental results. 
A semi-flexible chain is characterized by $L_{\mathrm{c}} \gg q \gg a$, where $L_{\mathrm{c}}$ is the contour length of the chain and $a$ is the monomer length. These two inequalities express the fact that the chain is flexible at large length scales and rigid at small scales. Let us now put such a chain in the vicinity of a non-adsorbing solid wall and suppose that it contacts the wall at a finite number of points. The inequality $L_{\mathrm{c}} \gg q$ ensures that the probability of the end monomers to be contact points is very small. On the other hand, the inequality $q \gg a$ introduces restrictions on the local curvature of the chain. In particular, around one contact point the radius of curvature cannot exceed $q^{-1}$. Therefore, if one monomer is near the wall, this implies that $q / a$ monomers are also in its vicinity. This is shown schematically in figure 1 . If we describe the chain through a Kuhn picture with freely-jointed segments of length equal to twice the persistence length, configuration la is permitted while configuration $1 \mathrm{~b}$ is forbidden. Here we suppose that the wall exerts no influence on the persistence length of the chain. The opposite case has been considered by Odijk in a somewhat different context of semi-flexible chains trapped in a cylindrical pore of diameter shorter than the natural persistence length for an unbounded chain [10]. We feel however that such deformations of the chain backbone conformation require tremendous elastic energies and that generates a force which in our case of a semi-infinite medium will tend to repel the chain from the wall.

Once we have realized that the conformation of a semi-flexible chain near a wall should obey the representation of figure $1 \mathrm{a}$, we can calculate the monomer concentration profile through a simple argument. Indeed each statistical segment of end-to-end distance $2 q$, containing $2 q / a$ monomers, can be replaced by a flexible subchain or blob with the same end-to-end distance, $\left\langle L^{2}\right\rangle_{\text {blob }}^{1 / 2}=2 q$ (it is obvious that the number of monomers contained in such a blob is larger than $2 q / a$, but we can always keep the mass inside one blob constant by renormalizing the monomer weight). The semi-flexible chain of freely-jointed straight segments is thus replaced by an uncorrelated sequence of $L_{\mathrm{c}} / 2 q$ blobs, each containing an ideal flexible sub-chain. When the number of blobs is small, it is customary to assume that the chain obeys Gaussian statistics. Therefore the end-to-end distance of this virtual chain is written as :

$$
\left\langle L^{2}\right\rangle_{\mathrm{F}}^{1 / 2}=\left(L_{\mathrm{c}} / 2 q\right)^{1 / 2} 2 q=\left(L_{\mathrm{c}} 2 q\right)^{1 / 2} .
$$

Let us recall that for a Kratky-Porod chain of curvilinear length $L_{\mathrm{c}}$ and persistence length $q$, the end-to-end distance $\left\langle L^{2}\right\rangle_{\mathbf{K P}}^{1 / 2}$ is written as [8] :

$$
\left\langle L^{2}\right\rangle_{\mathbf{K P}}^{1 / 2}=\left[2 q L_{\mathrm{c}}-2 q^{2}\left(1-\mathrm{e}^{-L_{\mathrm{c}} / q}\right)\right]^{1 / 2} .
$$

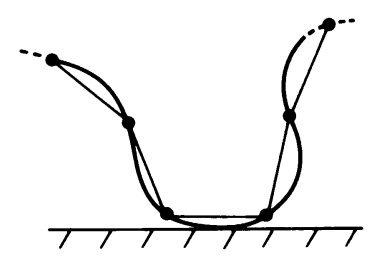

a)

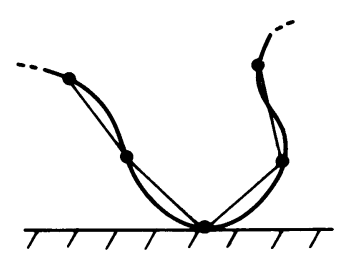

b) forbidden

Fig. 1. - Semi-flexible chain in the vicinity of a solid, non-adsorbing, wall. The chain is represented as a freely-jointed chain of segment lengths equal to twice the persistence length $q$. The conformation shown in (a) is compatible with the fact that if there is a monomer at the wall, the local chain rigidity implies that there is $2 q / a$ such monomers. The conformation shown in (b) is forbidden according to this rule. 
That is to say, for $L_{\mathrm{c}} \gg q$,

$$
\left\langle L^{2}\right\rangle_{\mathrm{KP}}^{1 / 2} \simeq\left(L_{\mathrm{c}} 2 q\right)^{1 / 2}
$$

and the two results are equivalent.

As shown in figure 2, we can now replace the actual semi-flexible chain in contact with the solid by its corresponding flexible chain in contact with a fictive wall located at a distance $z=-D$ $\simeq-2 q$. Any configuration of the semi-flexible chain which contributes to the depletion layer with the wall at $Z=0$ can be associated in the same manner to a configuration of the virtual chain with this fictive wall.

Starting from a theoretical profile $\Phi_{\mathrm{F}}(Z)$ for a flexible chain, we can deduce the depletion layer profile $\Phi_{\mathrm{SF}}(Z)$ for a semi-flexible chain through the relation

$$
\Phi_{\mathrm{SF}}(Z)=\Phi_{\mathrm{F}}(Z+D) \text {. }
$$

The concentration profile $\Phi_{\mathrm{F}}(Z)$ has been numerically calculated by Casassa [11] for isolated ideal chains. His result is very close to the afore-mentioned $\tanh ^{2}(Z)$ profile established in the semi-dilute regime and for good solvent conditions, and we find in both cases the same $Z^{2}$ dependence near the wall. The minute differences between the two models would be extremely hard to detect experimentally. Thus, for sake of simplicity, we choose to describe the flexible profile through the very simple expression :

$$
\Phi_{\mathrm{F}}(Z)=\Phi_{\mathrm{b}} \tanh ^{2}\left(\frac{Z}{R_{\mathrm{G}} \sqrt{2}}\right)
$$

which yields, for a semi-flexible chain :

$$
\Phi_{\mathrm{SF}}(Z)=\Phi_{\mathrm{b}} \tanh ^{2}\left(\frac{Z+D}{R_{\mathrm{G}} \sqrt{2}}\right) .
$$

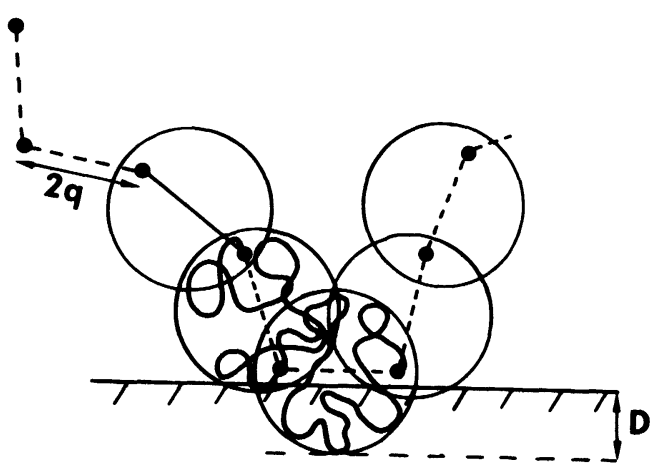

Fig. 2. - Same as figure 1. The dotted lines correspond to the Kuhn representation with freely-jointed segments of length $2 q$. The wiggling solid lines represent the virtual flexible sub-chains associated to each statistical unit. The circles indicate the spatial extension of each of these blobs (see text for details). This representation suggests that the semi-flexible and flexible cases are equivalent when the wall position is shifted by $Z=-D$. 
For $Z=0$, equation (5) predicts a finite monomer concentration at the solid surface

$$
\Phi_{\mathrm{SF}}(0) \simeq \Phi_{\mathrm{b}}\left(D / R_{\mathrm{G}}\right)^{2} .
$$

Note the quadratic dependence of $\Phi_{\mathrm{SF}}(0)$ on $D$ and therefore on the persistence length $q$.

The profile predicted by equation (5) can be compared to the experimental data previously obtained on aqueous solutions of xanthan in contact with a fused silica surface [2]. In the EWIF method, we always measure the ratio between the fluorescence emitted by the polymer solution of interest and the fluorescence emitted by a reference solution in which there is no depletion layer. This ratio $R$, is then plotted as a function of the inverse penetration length $\Lambda^{-1}$ for the optical evanescent wave probing the solution. The origin of distances is taken on the wall. The data points represented in figure 3 are for a $96 \mathrm{ppm}$ dilute solution. The solid line corresponds to the best fit with the theoretical profile. The agreement is excellent over the whole range of $\Lambda$, namely from $\infty$ down to $75 \mathrm{~nm}$. The parameters of the fit are $D$ and $R_{\mathrm{G}}$. We obtain $D=100$ $\pm 10 \mathrm{~nm}$ and $R_{\mathrm{G}} \sqrt{2}=255 \pm 15 \mathrm{~nm}$. Our extrapolation distance $D$ is in perfect agreement with the idea that $D$ correspond to the statistical length $2 q$ since the persistence length $q$ is known to be $50 \pm 2 \mathrm{~nm}$ [4]. On the other hand, the radius of gyration $R_{\mathrm{G}}$ for xanthan chains can be calculated from the Kratky-Porod formula using $R_{\mathrm{G}}=\left\langle L^{2}\right\rangle_{\mathrm{KP}}^{1 / 2} / \sqrt{6}$. For chains of molecular weight $2 \times 10^{6}$, we have $L_{\mathrm{c}}=1800 \mathrm{~nm}$. Therefore $\left\langle L^{2}\right\rangle_{\mathrm{KP}}^{1 / 2}=420 \mathrm{~nm}$ and $R_{\mathrm{G}}=170 \mathrm{~nm}$. Our experimental value of $180 \mathrm{~nm}$ is therefore in excellent agreement with this calculation.

On the whole, it is therefore clear that the present model provides a much more satisfactory description of the data than any of the previous ones. Another important parameter is the value of the concentration, $\Phi_{\mathrm{SF}}(0)$, right at the wall. Injecting the fitted values for $D$ and $R_{\mathrm{G}}$ into equation (6) we find $\Phi_{\mathrm{SF}}(0) \simeq 0.14 \Phi_{\mathrm{b}}=14 \mathrm{ppm}$. This surface concentration is by no means negligible compared to the bulk value of $96 \mathrm{ppm}$. It would be very interesting to measure this concentration directly by fluorescence resonance energy transfer between donors located on the wall and acceptors covalently attached to the chains. Contact angle measurements of sessile drops of the polymer solution should also give the same information. For semi-flexible chains the surface excess defined as $\Gamma=\int_{0}^{\infty}\left[\Phi(z)-\Phi_{\mathrm{b}}\right] \mathrm{d} z$ is diminished roughly by $D \Phi_{\mathrm{b}}$ relative to that of flexible coils. This is a large effect ( $39 \%$ in the present case) and the decrease in surface tension should be easily observable.

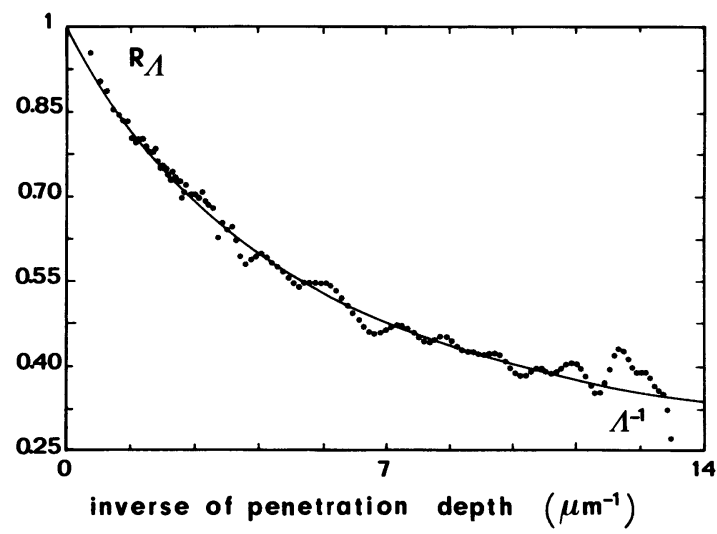

Fig. 3. - Fluorescence intensity ratio $R=I_{\mathrm{F}}^{\mathrm{pol}} / I_{\mathrm{F}}^{\text {ref }}$ versus the inverse of the penetration depth $\left(\Lambda^{-1}\right)$ of the evanescent wave. Xanthan polymer concentration $=96 \mathrm{ppm}$. The solid line corresponds to the best fit with the present model. 
To conclude, we have presented a conjecture allowing us to calculate the complete depletion layer profile in the case of semi-flexible chains, which is likely the most general case in polymer solutions. The functional form is analogous to the one already known for flexible chains, but with a virtual wall located at $Z=-D$, where $D$ is an extrapolation distance of the order of the chain persistence length. This new profile provides an excellent agreement with earlier experimental results on xanthan solutions. The numerical values obtained from the fit for both the persistence length and the radius of gyration of the chain correlate well with independent measurements of the chain properties in dilute bulk solution. The model predicts also a nonzero monomer concentration right at the wall. A direct measurement of the surface concentration would provide a definite check of the theory. Such experiments are currently underway in our laboratory.

\section{Acknowledgments.}

We thank P. G. de Gennes, J. F. Joanny and D. Andelman for their interest in this problem. Our work has been supported by an A.T.P. " Polymères aux Interfaces " from the C.N.R.S. One of us (D. Ausserré) wishes to acknowledge personal financial support from Elf-Aquitaine and constant encouragement from D. Lourdelet and P. Girard.

\section{References}

[1] See for instance High Technology 4 (1984) 66.

[2] Ausserré, D., Hervet, H. and Rondelez, F., Phys. Rev. Lett. 54 (1985) 1948.

[3] Allain, C., Ausserré, D. and Rondelez, F., Phys. Rev. Lett. 49 (1982) 1694.

[4] Muller, G., Lecourtier, J., Chauveteau, G. and Allain, C., Makromol. Chem. Rapid Commun. 5 (1984) 203.

[5] Wellington, S. L., A.C.S. Polym. Preprints 22 (1981) 63.

[6] Johnny, J. F., Leibler, L. and De Gennes, P. G., J. Polym. Sci., Polym. Phys. Ed. 17 (1979) 1073.

[7] Auvray, L., J. Physique 42 (1981) 79.

[8] Yamakawa, H., Modern Theory of Polymer Solutions (Harper and Row, New York) 1971.

[9] Ausserré, D., Hervet, H. and RondelEZ, F., submitted to Macromolecules (1985).

[10] ODJIK, T., Macromolecules 16 (1983) 1340.

[11] CASASSA, Macromolecules 17 (1984) 601. 